\title{
Benoist Pierre, Le Père Joseph. L'Éminence grise de Richelieu
}

Paris, Ếditions Perrin, 2007, 476 p.

Jean-Pascal Gay

\section{(2) OpenEdition}

\section{Journals}

Édition électronique

URL : http://journals.openedition.org/assr/23552

DOI : $10.4000 /$ assr.23552

ISSN : $1777-5825$

Éditeur

Éditions de l'EHESS

Édition imprimée

Date de publication : 31 décembre 2011

Pagination : 258

ISBN : 9782713223273

ISSN : 0335-5985

\section{Référence électronique}

Jean-Pascal Gay, «Benoist Pierre, Le Père Joseph. L'Éminence grise de Richelieu », Archives de

sciences sociales des religions [En ligne], 156 | octobre-décembre 2011, document 156-92, mis en ligne le 16 février 2012, consulté le 21 septembre 2020. URL : http://journals.openedition.org/assr/23552 ;

DOI : https://doi.org/10.4000/assr.23552

Ce document a été généré automatiquement le 21 septembre 2020.

(c) Archives de sciences sociales des religions 


\section{Benoist Pierre, Le Père Joseph. L'Éminence grise de Richelieu}

Paris, Ếditions Perrin, 2007, 476 p.

Jean-Pascal Gay

\section{RÉFÉRENCE}

Benoist PIERRE, Le Père Joseph. L'Éminence grise de Richelieu, Paris, Ếditions Perrin, 2007, $476 \mathrm{p}$.

1 Le second ouvrage de Benoist Pierre, paru en 2007, est une biographie à la croisée de plusieurs sillons de recherche : histoire du politique et des cultures politiques, histoire de la diplomatie et du renseignement, histoire sociale de la noblesse et des élites de gouvernement et histoire religieuse. Basée sur des sources nombreuses et diverses (même si l'on pourrait regretter qu'elle néglige un peu les sources romaines) et remarquablement écrite, cette biographie de François Le Clerc de Tremblay, devenu dans l'ordre capucin le Père Joseph de Paris, l'« éminence grise » de Richelieu, s'impose comme une remarquable réussite malgré la difficulté de concilier des historiographies et des problématiques hétérogènes à l'intersection des champs que le récit biographique devait mobiliser pour demeurer historiquement heuristique.

2 On voudrait ici se concentrer plus précisément sur ce qui dans cette étude concerne le religieux. La biographie du Père Joseph proposée ici est une biographie à thèse. À bien des égards, elle s'inscrit clairement dans la continuité de La Bure et le Sceptre (2006), où l'auteur en s'appuyant sur l'exemple des relations multiples entre la congrégation cistercienne des Feuillants et la monarchie, montrait que, s'il y a bien eu une autonomisation du politique au tournant des $\mathrm{XVI}^{\mathrm{e}}$ et $\mathrm{XVII}{ }^{\mathrm{e}}$ siècles, cette autonomisation pouvait être qualifiée d'autonomisation "religieuse", en ce qu'une rationalisation proprement religieuse du politique y a fortement contribué. Un idéal et une mystique de sujétion religieuse pouvaient être mis au service des prétentions absolutistes des 
princes, laquelle, dans le deuxième tiers du siècle, pouvait même se résoudre en une sainteté civile, une adhésion véritablement religieuse au catholicisme d'État.

3 En étudiant la figure exemplaire du Père Joseph, artisan de la construction de ce catholicisme d'État, c'est bien sur la complexité et la plurivocité des relations entre politique et religion, que Benoist Pierre entend revenir: la vie du capucin semble épouser la chronologie de ces relations, passant d'une aspiration universaliste à la réconciliation pour surmonter les troubles passés (personnels et collectifs) à une véritable religion royale, qui voit dans le service du Roi Très-Chrétien, l'espoir providentiel de la Chrétienté.

4 Ce fil sous-tend la présentation chronologique de la vie du religieux devenu serviteur de la construction de l'État moderne. Ainsi la première partie qui suit le cheminement social, familial et religieux de François Le Clerc jusqu'à son entrée dans l'ordre capucin en 1599, peint un homme traversé par les tensions sociales et religieuses du temps : une famille prise entre un éthos d'épée et la nécessité d'alliance et même de véritable passage à la robe, une famille divisée par les conflits confessionnels, mais aussi par ceux qui opposent les catholiques autour de leur rapport au politique, une famille marquée par les conflits perpétuels que produit le risque d'un déclassement social, enfin une spiritualité caractérisée par une inquiétude mystique encore habitée par une angoisse eschatologique. La vocation du Père Joseph, que l'auteur lie à l'ensemble de ces tensions, apparaît alors non plus seulement comme une fuite du monde, mais plus spécifiquement comme une fuite des « divisions » du monde.

5 En retour, la première période de l'activité de celui qui est désormais le père Joseph, de 1600 à son entrée au service de Richelieu en 1624, est caractérisée par un principe d'action : une recherche plurielle et universelle d'unité. L'auteur en suit la trace et les évolutions dans l'action apostolique du capucin auprès de safamille, dans les spécificités de sa doctrine par rapport à celle de son maître Benoît de Canfield, dans la direction de la jeune congrégation des Filles du Calvaire, mais aussi dans son action en faveur du projet de croisade de la Milice Chrétienne. L'échec de ce projet aurait incidemment contribué à confirmer l'idée que la prééminence du Roi de France était la condition d'un dépassement des divisions de la Chrétienté, au service de laquelle le Père Joseph mettait aussi une mystique ouvrant la voie à un principe d'autorité de type absolutiste.

6 Enfin, dans la période de pleine activité du Père Joseph, la contradiction entre la vocation première du religieux et son état de vie théorique d'une part, et la vie d'un serviteur de l'État d'autre part, est résolue par une radicalisation du modèle spirituel du Père Joseph et par l'intégration de l'activité politique à un idéal de vie mixte.

7 C'est peut-être cependant à partir de ce dernier point qu'apparait une des difficultés auxquelles s'est heurté l'auteur. À la figure historique d'un religieux dans lequel s'incarne une aspiration à dépasser les divisions du siècle et qui devient à la fois acteur et exemple de ce dépassement correspond la démarche historienne de l'auteur qui cherche à montrer la cohérence profonde de cette vie exemplaire des évolutions du catholicisme français du siècle. Ainsi, justement, à propos du risque d'incompatibilité entre les conséquences de l'activité politique du Père Joseph sur son mode de vie et son état régulier, l'auteur récuse explicitement l'idée que le religieux ait pu séparer son rôle politique et sa spiritualité. Il considère au contraire que le Père Joseph a placé au cœur de sa spiritualité la catégorie de "vie mixte », intégrant son service de l'État à un modèle de vie héroïque. Il n'est pas certain cependant que le remploi de cette catégorie 
théologique traditionnelle par le Père Joseph dans sa correspondance avec les Filles du Calvaire puisse être lu comme englobant véritablement le service politique du souverain; du moins un tel usage ne pourrait-il fonctionner sans difficulté. On a là un exemple, parmi d'autres, d'une difficulté qui traverse cette relecture de la vie du Père Joseph : quelles sont les ressources linguistiques et culturelles qui permettaient (ou ne permettaient pas) au P. Joseph d'affronter les contradictions auxquelles sa vie, mais aussi les tensions internes au catholicisme du temps, le confrontaient ? C'est d'autant plus frappant que d'autres réagissaient différemment à de telles contradictions, et que ces dernières étaient aussi au cœur de certaines évolutions culturelles dans le monde catholique. La nécessité de gérer les impératifs contradictoires du politique et du religieux est une question qui se posait à tous et qui ne trouvait pas de réponse uniforme.

Il n'est par ailleurs pas indifférent que cette difficulté porte en réalité aussi sur l'usage de l'écrit par le capucin. L'auteur décrit un religieux constamment appliqué au travail d'écriture : correspondance, mémoires et projets politiques et diplomatiques, écrits polémiques, écrits spirituels, etc., sans jamais vraiment interroger cette activité d'écriture et les modalités de son efficacité, tant dans l'action politique et religieuse que dans la gestion des contradictions individuelles et collectives auxquelles le Père Joseph est exposé. L'aspiration à la cohérence et à l'unité souvent présente dans ces écrits devient alors souvent le signe même de l'unité de vie dont le biographe fait le cœur de l'exemplarité de François Le Clerc du Tremblay. Au final, cet excès de cohérence et d'exemplarité ne va pas non plus sans risque de sous-estimation des tensions à l'œuvre dans le catholicisme français mais surtout dans sa culture confessionnelle au lendemain des guerres de religion.

Il n'en demeure pas moins que cette biographie déconstruit efficacement le mythe fortement enraciné qui faisait du Père Joseph l'exemple d'un mystique dévoyé en politique et permet aussi de repenser l'action de Richelieu et de son entourage de ce point de vue. Ce que l'auteur offre, c'est non seulement le dépassement de la dichotomie entre raison d'État et raison d'Église, mais encore le moyen de penser la contribution du discours, de l'écriture et de l'engagement mystique aux transformations politiques du siècle. 